\title{
Dipper: A Dynamically Transitioning Aerial-Aquatic Unmanned Vehicle
}

\author{
Friedrich M. Rockenbauer*, Simon L. Jeger*, Liberto Beltran, Maximilian A. Berger, Marvin Harms, \\ Noah Kaufmann, Marc Rauch, Moritz Reinders, Nicholas Lawrance, Thomas Stastny, Roland Y. Siegwart \\ Autonomous Systems Lab, ETH Zurich, Switzerland
}
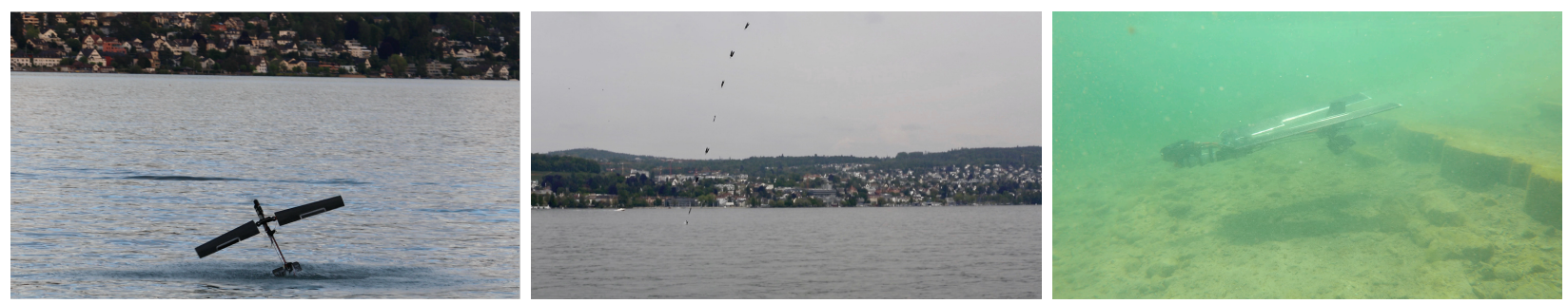

Fig. 1: Dipper platform in different configurations (from left to right): (i) emersion phase, (ii) sequential image during dive-in and (iii) underwater.

\begin{abstract}
The locomotion for many modern robotic systems is optimized for a single target domain - aerial, surface or underwater. In this work, we address the challenge of developing a robotic system capable of controlled motion in air and underwater. Further, we explore the particular challenge of dynamic transitions between air and water. We propose Dipper, an aerial-aquatic hybrid vehicle. Dipper is a lightweight fixed-wing unmanned aerial vehicle (UAV) with actively swept wings. The bio-inspired system is not only capable of maneuvering efficiently during flight and underwater, but can also perform dynamic aerial-aquatic transitions. We describe the design, construction and testing of the Dipper prototype, and demonstrate repeatability and robustness especially during the transition phases.
\end{abstract}

\section{SUPPLEMENTARY MATERIAL}

A video showing a full operation cycle can be found at https: //youtu.be/KV90qqnhHb0. Further information and additional material can be found at https://www.dipper.ethz.ch. [

\section{INTRODUCTION}

In nature, locomotion strategies are usually highly optimized for one type of environment. But the animal domain has some members that seem to have mastered the transition between those different environments. The northern gannet for example, a bird of $2 \mathrm{~m}$ wingspan and $3 \mathrm{~kg}$ body weight, plunge dives $10 \mathrm{~m}$ under water, hitting the water surface at up to $100 \mathrm{~km} / \mathrm{h}$, to hunt fish [5]. Another example are penguins. Unable to fly, and with limited mobility on land, penguins are nevertheless very agile underwater, with speeds up to $5.3 \mathrm{~m} / \mathrm{s}$ allowing them to jump from water to land [3].

A combination of the abilities of these two species would allow efficient travel in the air and underwater as well as a smooth and dynamic transitioning between both media. The

\footnotetext{
* Authors contributed equally. Corresponding author contact: rockenbfeethz.ch
}

resulting system would offer high dynamic range which is independent of connected water regions, as typical for UAVs, as well as the stability, robustness and exploration capability, as typical for unmanned underwater vehicles (UUVs).

Modern UAVs can be split into three categories based on their operation principle: fixed-wing aircraft, rotor craft and lighter than air vehicles. Lighter than air vehicles are difficult to submerge under water because of their high buoyancy. In comparison, rotor craft tend to have higher densities, but suffer from limited range in both media and dynamic capabilities underwater [2, 13]. In this work we focus on a fixed-wing design, based on flight efficiency and relative simple actuation concept.

The main challenge for a vehicle operating in both air and water is the large difference in density between the two media. On one hand, the conductivity and corrosive properties in combination with the high loads subjected to the vehicle by water require the system to be sealed, robust and have minimized displaced volume to reduce buoyancy. On the other hand, fixed-wing flying systems require large lifting surfaces and a minimized mass to remain airborne. This leads to a contradicting set of requirements.

A system which can operate dynamically in the air, during transition phases and underwater with the necessary robustness and capability to carry at least small payloads has yet to be developed. Our proposed solution is a fixed-wing UAV with wing-folding capabilities, powered by a single electric motor (Fig. 2). The system is agile and efficient in the air as well as dynamic underwater achieved by an optimized propulsion system and actuation concepts for both areas of operation. The folding wing concept provides a reduced cross-sectional area for the dive-in phase and a shift of the center of lift/buoyancy with respect to the center of mass for ideal trim in all operation modes. With a wingspan of $2.1 \mathrm{~m}$ and a take-off weight of 
$3.1 \mathrm{~kg}$ the system is large enough to carry small payloads and withstand wind, waves and weather to a certain point and small enough to be easily transported and hand-launched.

\section{RELATED WORK}

While robotic locomotion in both air and water have been independently well-studied, there are limited existing approaches to operating in both air and underwater with a single platform.

One of the primary challenges is that, while conceptually similar, the requirements for rotor-based propulsion systems are different for efficient operation in air and water. Due to the high density of water and the effects of cavitation, underwater propellers tend to be slower-rotating and with higher projected area ratios than aerial propellers. Further, the transition between air and water, especially emersion from water, requires a rapid change in operating mode of the propulsion system.

Early research in hybrid aerial-aquatic vehicles focused on multirotors, because (aside from longer-term degradation due to corrosion) the brushless motors used in most aerial multirotors also work underwater. Drews et al. [4] propose and simulate a design based on a typical quadrotor form factor that has axially-aligned air/water propeller groups. The vehicle is partially submerged during transition, so that while resting on the surface of the water the underwater propellers are submerged but the aerial propellers remain in air. A similar concept is explored and demonstrated in [8], except the propellers are the same and can be used in air and underwater. The motors transition between high- and low-speed modes at the surface of the water, with the lower set still underwater while the upper set operate in air for emersion. This work provided the first public demonstration of multirotor transition between aerial and underwater motion. Alzu'bi et al. [1] evaluate the efficiency and effectiveness of a single propeller on each arm for both underwater and aerial operation, but do not demonstrate transition between media. Our work uses a similar approach to [4] with separate, dedicated underwater and aerial propellers, but to minimise weight we use only a single motor and a unique clutch system.

More recent work has explored the use of winged designs for hybrid air/water vehicles, largely inspired by the capabilities of diving birds like the gannet. Transition between media are particularly difficult due to the high difference in operating speeds in air and water. Plunge diving birds partially fold their wings to reduce contact area and impact force when diving into the water [7]. This also provides a lower-drag shape, and these birds use their legs and wings for controlled motion underwater. A variety of hybrid designs, both natural and synthetic, are explored in [9]. The same work proposes the AquaMav concept, a folding-wing design that proposes using a pressurized gas cartridge to power a water-jet thruster for the emersion transition from underwater to aerial flight. A hybrid propulsion system is demonstrated in [14], using an epicyclic gearbox with a single motor and propeller for propulsion underwater and in air, but with insufficient power for transition. Continued development in [10] demonstrated the use of a compressed gas cartridge for water emersion, and finally in [11] with a $34 \mathrm{~g}$ water-jet thruster based on calcium carbide combustion capable of producing over $20 \mathrm{~N}$ of thrust for transition from underwater to aerial flight. The Dipper design combines a folding wing design (as in [9]) with a single electric propulsion system (as in [14]), but with sufficient power for emersion, eliminating the need for a separate emersion propulsion system.

Another significant development in underwater/aerial hybrid vehicles was the EagleRay platform [15]. EagleRay also uses shared motor and propeller with a gearbox that provides different underwater and aerial motor speeds and torques. To avoid the problem of rapid switching required for dynamic emersion, EagleRay is positioned in a vertical (nose up) orientation at the water surface, allowing the propeller to operate in air and provide sufficient thrust to climb out of the water. The EagleRay wings do not fold for immersion, but rather the vehicle lands in a slower, more controlled fashion in the water. Hollow wings that drain during climbout provide near-neutral buoyancy underwater. A prior version of the EagleRay platform contains an independent propulsion system for water and air operation [12]. The system includes two motors, each with a separate electronic speed controller (ESC), which propel an underwater screw in the back and an aerial propeller in the front. Dipper uses a similar approach to EagleRay for water egress, but our folding wing design and rapid motor switching allows our propulsion system to provide enough power and underwater speed for dynamic emersion. Our folding-wing design also allows for plunge-dive immersion, and rapid transition to underwater operation.

\section{SySTEM OVERVIEW}

\section{A. General Overview}

The overall goal of the Dipper system is to demonstrate an aerial/underwater hybrid vehicle capable of repeatable dynamic water ingress and egress. Drawing inspiration from previous research, Dipper is based on a fixed-wing aerial platform, with folding wings for ingress diving. We propose and demonstrate the use of a novel hybrid propulsion system that provides sufficient thrust for underwater motion, and then switches to an aerial tractor propeller during egress. The thrust required necessitates a dedicated propeller (underwater screw) for underwater operations, and flight requires a second specialized aerial propeller. To minimize weight, the propellers share a motor that can be driven in both rotation directions and uses a freewheel clutch system that engages only one of the propellers at a time based on the rotation direction.

The Dipper vehicle prototype, as displayed in Fig. 2, was developed and tested, demonstrating the capability to complete an operational cycle consisting of launch, flight, ingress, underwater cruise and return to flight (Fig. 11). Several manual and automatic control modes exist for operation in air, whilst the underwater behaviour consists of automatic trajectory following. 


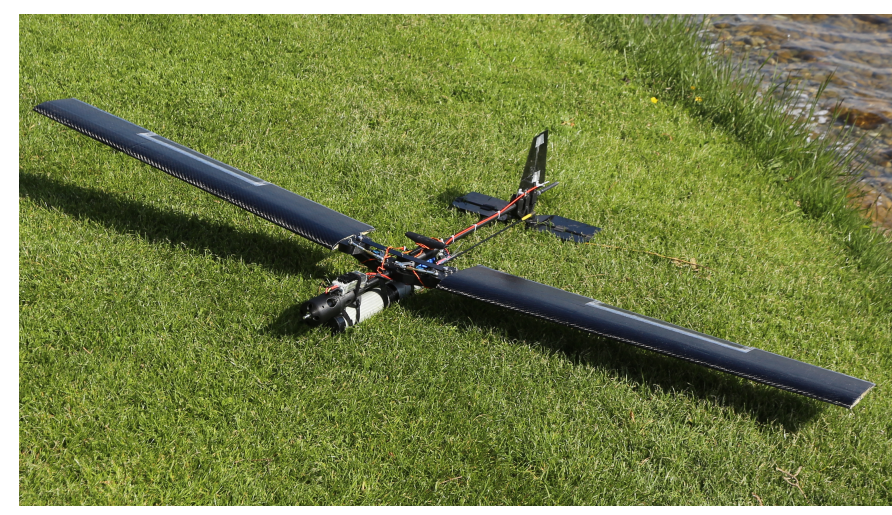

Fig. 2: Prototype in flight configuration.

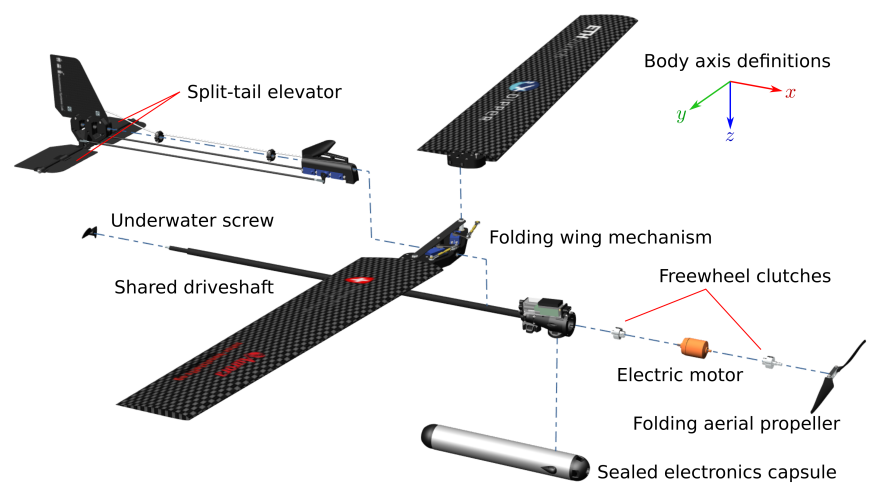

Fig. 3: Exploded view of Dipper prototype.

The vehicle weighs $3.1 \mathrm{~kg}$ and features a $2.1 \mathrm{~m}$ (at maximum extension) variable-sweep wing. A single $1000 \mathrm{~W}$ electric motor can selectively drive either a folding propeller at the front, or an underwater screw at the rear. The wings have independent sweep angle actuators, and feature an 87 degree range of motion. Tables $\Pi$ and $\Pi$ provide a list of the overall system capabilities and properties of the Dipper vehicle. The maximum achievable depth was a chosen design parameter and was supported by calculations. Measured depths of over $5 \mathrm{~m}$ have been repeatedly achieved during testing.

To reach level flight, the vehicle can either take off vertically out of the water or be hand-launched by the operator. In flight configuration, the wings are swept fully forward and thrust is provided by the conventional air propeller. Control is provided by traditional elevator, rudder and aileron control surfaces. For water entry, the wings transition into their fully swept

\begin{tabular}{ll}
\hline Stall speed & $36 \mathrm{~km} / \mathrm{h}$ \\
Cruise speed & $50 \mathrm{~km} / \mathrm{h}$ \\
Max. speed (level flight) & $110 \mathrm{~km} / \mathrm{h}$ \\
Max. dive speed (wings folded) & $130 \mathrm{~km} / \mathrm{h}$ \\
Max. dive height & $150 \mathrm{~m}$ \\
Flight endurance & $4 \mathrm{~min}$ \\
Flight range & $5 \mathrm{~km}$ \\
Max. underwater speed & $3 \mathrm{~m} / \mathrm{s}$ \\
Underwater endurance & $8 \mathrm{~min}$ \\
Underwater range & $500 \mathrm{~m}$ \\
Max. depth (calculated) & $10 \mathrm{~m}$ \\
Max. depth (tested) & $5 \mathrm{~m}$ \\
\hline
\end{tabular}

TABLE I: System capabilities of the Dipper platform.

\begin{tabular}{ll}
\hline Length & $1.16 \mathrm{~m}$ \\
Weight & $3.1 \mathrm{~kg}$ \\
Motor Power & $1000 \mathrm{~W}$ \\
Wingspan & $2.1 \mathrm{~m}$ \\
Width (when folded) & $400 \mathrm{~mm}$ \\
Root Chord & $200 \mathrm{~mm}$ \\
Taper Ratio & 0.8 \\
Airfoil & AG35 \\
\hline
\end{tabular}

TABLE II: Physical characteristics of the Dipper prototype.

configuration allowing slower deceleration during impact and reduce drag underwater. The vehicle follows an unguided ballistic trajectory into the water, engaging the underwater screw shortly before impact. Underwater roll and pitch authority is achieved by independently manipulating the left and right elevators. The vehicle is positively buoyant and consequently requires a minimal forward velocity to maintain depth and attitude. Transitioning from underwater maneuvering to flight is accomplished by gradually sweeping the wings forward underwater, and entering a nearly vertical orientation to the water surface. When the vehicle detects the water surface, it automatically engages the propeller and utilizes a thrust-toweight ratio greater than one to perform a vertical take-off.

\section{B. Design Principles}

The design of a multi-domain vehicle introduces significant challenges due to competing design principles when operating in different media. Developing a vehicle that is light enough for flight yet still able to submerge requires minimizing displaced volume. Correspondingly, no aerodynamic fairings are used, the volume of the electronics capsule is reduced by directly immersing waterproof electronics wherever possible and the elevators and rudder are constructed from flat carbon sheets. To promote rapid design evolution, the vehicle design is based around the propulsion system (consisting of propeller, freewheel clutches, motor, driveshaft and underwater screw). All vehicle components are attached to a common carbon shaft that encloses the driveshaft, enabling independent design evolution of the sub-assemblies.

\section{Hardware}

1) Propulsion System: The critical mission mode when designing the propulsion system for an aquatic UAV is the transition from underwater into flight. Existing aquatic UAVs dive out of the water either dynamically by accelerating underwater and jumping out of the water [10, 11] or statically by vertically taking off from the water surface [1, 4, 15]. Those two strategies are reflected in the different propulsion designs.

Vehicles with a fixed propeller-motor combination for propulsion in both media face a trade-off of efficiency underwater versus efficiency in the air. Due to the fundamental difference in density of water and air, using just one propeller implies that the necessary rotation speed underwater is much smaller than in flight. An electric motor, therefore, operates at least in one of the two media at low efficiency [14]. Since low efficiency during flight leads to high power consumption 
and a short operation time, most existing aquatic UAVs favor aerial efficiency over aquatic efficiency. Consequently, they do not reach high enough velocities underwater that would allow for a jumping dive-out.

One focus of the presented project was to minimize the duration of the transition phase and the primary objective in the design process of the propulsion system was to dive-out dynamically. This goal is motivated by the higher robustness against environmental influences (e.g. waves or side wind) when transitioning from the water to the air with higher velocity. As systems that use an aerial propeller for underwater propulsion do not reach the necessary speed underwater that would allow for a jumping dive-out, we aimed for an alternative propulsion system. Nonetheless, we decided against using gas thrusters as it is complex to design this system to be reusable - although this has been done using combustion of solid calcium carbide [11]. Another consideration was selecting an impeller, as used in water scooters and jets, but different designs proved to be incapable of transitioning from water to the air due to the high difference in required rotation speeds. Alternatively, various designs containing a underwater screw were evaluated. The main advantage of a configuration with a separate propulsion system for each medium is the possibility to tune each system such that efficient propulsion is achieved in both media, possibly with only one motor. We decided against using two separate motors due to the additional weight added by the motor and associated electronics.

In a single motor system a controlled clutch is necessary to actuate each propeller independently. Operating the propeller with an externally actuated clutch would take advantage of the kinetic energy stored in the motor when accelerating during dive-out. This idea was not pursued further since externally actuated clutches capable of transmitting the required torques are too bulky and heavy. Inspired by a system that uses a variable transmission rate dependent on the rotation direction of the motor [14], we developed a system based on two freewheel clutches attached to one motor. Therefore either the underwater screw or the propeller is driven depending on the rotation direction of the motor. Since there are no additional actuators, the design adds little additional weight and complexity. This concept requires a complete stop and reacceleration phase of the motor to switch between water and air operation.

As shown in Fig. 4 freewheel clutches with opposite torque transmission directions are mounted on both sides of the motor. The front clutch is connected to the propeller, and the rear clutch is attached to the drive shaft, which drives the underwater screw. Plastic sliding bearings are added to increase stability. The final design includes a brushless DC motor with a maximum continuous power of $1000 \mathrm{~W}$ and $890 \mathrm{RPM} / \mathrm{V}(\mathrm{Kv})$ powered by a four-cell lithium battery $(14.8 \mathrm{~V})$ with a capacity of $2200 \mathrm{mAh}$. In air, the system is driven by a right-turning, folding propeller with 14 inch diameter and 9 inch pitch. Foldability is crucial to avoid damage during ingress and considerably lowers drag during underwater operation. This combination of motor and propeller

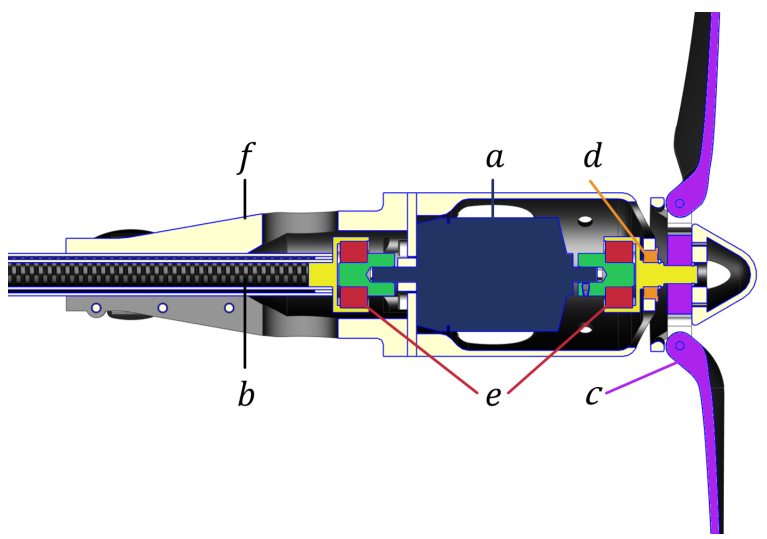

Fig. 4: Clip section of propulsion concept - a) motor (blue), b) drive shaft (carbon), c) propeller (violet), d) ball bearing (orange), e) freewheel clutch (red), f) housing (cream).

results in a maximum thrust to weight ratio of 1.4. This setup is the result of many tested motor propeller combinations evaluated on the provided thrust and the time required to change the rotation direction. Together with the underwater speed, those are the key parameters for a successful jumping dive-out. Especially the minimization of the switching time (the time required to transition from full speed in one direction to a thrust to weight ratio of 1 in the other spinning direction) was investigated extensively. The minimal switching time of the tested brushless DC motors, all attached to a $14.8 \mathrm{~V}$ battery and differing mainly in their $\mathrm{Kv}$ value (from $500 \mathrm{RPM} / \mathrm{V}$ to $1000 \mathrm{RPM} / \mathrm{V})$, were all within a range of 10 percent. The chosen motor and propeller combination reached a switching time of $0.3 \mathrm{~s}$ and provides a maximum thrust of $42.6 \mathrm{~N}$ in the air. The parameters of the underwater screw were mainly chosen by tests measuring the provided thrust underwater. The best results were achieved by a left-turning, $65 \mathrm{~mm}$ diameter, three-bladed underwater screw with a blade pitch of $34 \mathrm{~mm}$ that produced a peak thrust of $60 \mathrm{~N}$.

The presented propulsion system allows for efficient movement underwater and in air. The selected locomotion system provides a maximum speed of $3 \mathrm{~m} / \mathrm{s}$ underwater. In combination with a motor switching time of $0.3 \mathrm{~s}$, the configuration makes a jumping dive-out feasible. Compared to systems with just one propulsion system for both media, this concept has little additional complexity and weight.

2) Wing folding Mechanism: The water ingress exposes the structure and especially the wings to substantial forces, which are sought to be minimized by having a wing folding mechanism. Sweeping back the wings minimizes the cross sectional area for impact and hence the acting forces. It introduces novel possibilities of enhancing flight performance and new control strategies through direct wing sweep control as shown in our previous work [6].

The mechanism consists of two four-bar linkages, each actuated directly by a servo motor Fig. 5. The sweeping angle ranges from 0 to 87 degrees. The orientation of the actuating lever arm was chosen such that it reduces the necessary applied torque during regular flight. The mechanism makes use of spherical joints to ensure that mechanical tolerances 


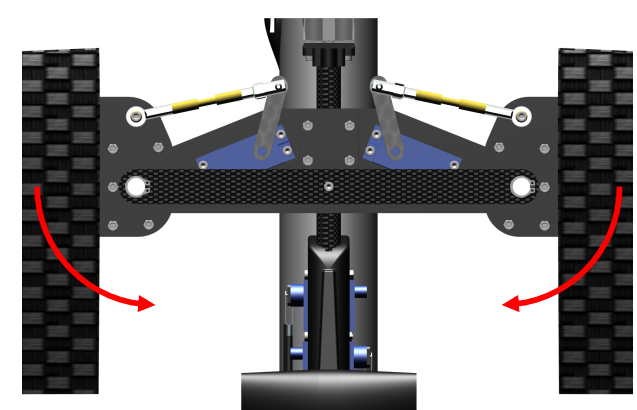

Fig. 5: Four-bar-linkage-mechanism to fold wings.

and deformations due to external forces do not inhibit the functionality. Following the modular approach of clamping the sub-assemblies to the central carbon rod makes it easy to adjust the position. This allows adjustments to the relative position of center of lift and center of gravity. The wings are mounted with retaining rings, which makes disassembling and replacement easy.

The choice of having two servo motors actuating each side separately is motivated by the drive train being placed in the middle of the structure and thereby obstructing the placement of single components on the axis of symmetry. Asymmetric placement is not favourable due to the size and weight of the servos. Although the upright orientation of the servos creates a larger target for fluid dynamic forces, it minimizes the number of links required for the mechanism.

The wings, being the largest closed volume of the overall structure, are the main source of buoyancy under water. When swept back, this creates a large torque on the mounting point around the pitch axis. This is countered by a retaining element set behind the bearings. This element was designed to only counter the forces pointing in negative $z$-direction in body frame (see Fig. 3). This keeps the system in a more controllable configuration during underwater maneuvers in a vertical orientation. The deflections during flight are less concerning, as the torque produced by the lift of the fully deployed wings can be countered by exploiting the symmetry and stiffening the structure with a rod.

3) Electronics: When designing the electronics for an aquatic UAV some unique challenges have to be faced. The components must be either individually waterproof or protected by a sealed compartment. This compartment must be compact to reduce cross sectional area, volume and therefore buoyancy. Placement of the electronic components is crucial in order to minimize electromagnetic interference. Furthermore, mass must be limited to enable dynamic dive-out and reduce the cruise speed of the vehicle during flight.

Fig. 6 shows the components chosen for this project. The largest in terms of weight and volume in the capsule is the battery. It powers the actuators through the ESC and is connected to a power module supplying the electronics with stabilized $5 \mathrm{~V}$. The commercial Pixhawk 4 is used as the flight management unit. It is connected to all actuators enabling control using pulse-width modulated (PWM) signals. To estimate the vehicle state during flight the Pixhawk has a built-in inertial measurement unit (IMU), and an external

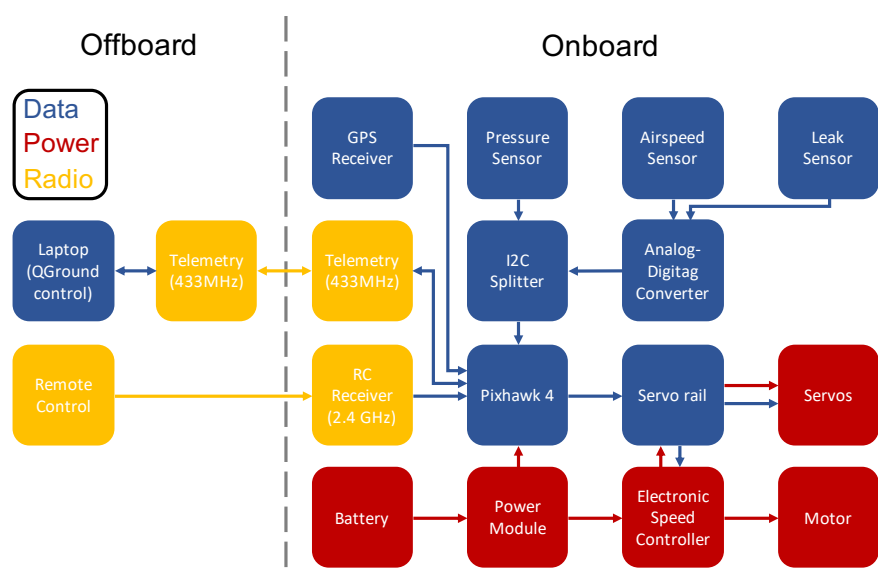

Fig. 6: Overview of primary electronic components.

GNSS receiver provides absolute position measurements.

While underwater, the state purely consists of the orientation and accelerations measured by the IMU and depth measured by a pressure sensor (TE MS5837-02BA). The pressure sensor output is also used in air, acting as a barometer to provide a better approximation of the current altitude. Furthermore, a leak-detection sensor is used to detect water entering the electronics capsule while submerged.

In flight the system uses two radio links to communicate with ground operators. A $2.4 \mathrm{GHz} \mathrm{RC}$ Receiver connects to the pilot console enabling manual control of the system in the air. Telemetry data can be sent and received using the MAVLink protocol over a $433 \mathrm{MHz}$ radio telemetry connection. Both only penetrate the water surface by about $10 \mathrm{~cm}$. Therefore the system has to operate underwater without input from the operator.

\section{Software}

1) Navigation and Position Control: The task of navigating depends strongly on the environment the system is operating in. Especially for sensors which are optimized for a limited set of operation conditions, the change of medium typically leads to reduced accuracy or inaccurate measurements. Therefore, the navigation strategy also had to be adopted for the different states described in Fig. 11

To exploit the sequential structure of the process, a position controller based on a nested state machine was implemented as shown in Fig. 7. The outer-most state machine has (aside from failure and recovery states) five main states: flight $(a)$, dive-in $(b)$, stabilize $(c)$, swim $(d, e)$ and dive-out $(f, g)$ using different levels of guidance (indicated in Fig. 11).

The flight state offers several operation modes mainly provided by the PX4 platform, starting from full manual control up to a pre-programmable mission mode (Fig. 7 only shows the manual flight mode for simplicity reasons). Typically all installed sensors including GNSS, IMU and magnetometer can be used during flight for localization and state estimation. During the free fall dive-in procedure no navigation action is taken to not interfere with a safe penetration of the water surface. Main focus lies on the vehicle attitude and wing folding. Since this process is highly dynamic, underwater 


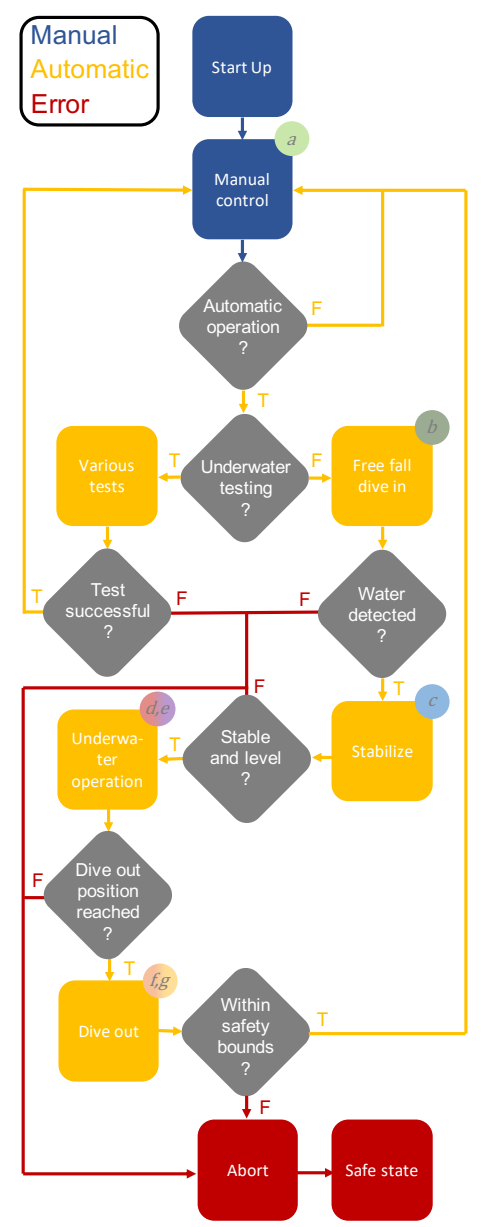

Fig. 7: Flow chart of the main state machine of the Dipper platform as it was used during all experiments.

operation starts with reestablishing a stable vehicle state in a horizontal orientation. The submerged vehicle has to operate with a heavily reduced sensor suite. First and foremost the lack of an absolute positioning system (GNSS) influences the estimation accuracy and limits the time the system can operate fully submerged. The state estimator mainly operates on IMU, magnetometer and pressure sensor data. To constrain the localization solution drift, the vehicle can surface to regain a global position estimate via GNSS and reestablish connection between the vehicle and ground station. The main focus of the position control algorithm under water is the target depth. Since the depth and vehicle attitude are the most important factors for a successful dive-out, the same control structure is used for the first part of the egress phase. During the short, unstable air time in this phase, navigation is of lower importance. Therefore, navigation can take over guidance when the system is back in level flight dependent on the desired operation mode in the air.

Due to the dynamic nature of the transition phases, fallback scenarios were implemented to ensure safe operation. The system is positively buoyant and will float on the water surface if not actuated. This was identified as the safest vehicle state and used as a target for recovery scenarios close to the water (e.g. unsuccessful dive-outs, errors underwater). When a prob- lem is identified (e.g. timeout, state out of limit), automatic procedures are terminated and an emergency dive-in including wing folding is initiated. As an additional safety measure, the emergency dive-in can be overruled by the operator.

2) Attitude Control: Automatic control is required to stabilize the vehicle attitude. Because of the different vehicle dynamics and control surfaces used in air and water, two different attitude controllers are used, one when submerged in water and another for aerial flight. In both cases, the controller is structured as a cascaded PID-controller. The modelling of the aerial dynamics was performed in a similar fashion to the approach shown in [6]. We used a rigid body model of the aircraft and the estimated aerodynamic forces to compute state derivatives that are then numerically integrated to obtain the full state trajectory. Since we obtained the aerodynamic forces from the Athena vortex lattice solver 1 , the model was not used for theoretical analysis but only to qualitatively evaluate flight behaviour in simulation. The standard rate-control structure for fixed wing aircraft from the PX4 platform 2 is used for attitude control during aerial flight. The basic mapping of the control signals to control surfaces is conducted in a traditional way, with roll and pitch body axis mapping to ailerons and elevator, respectively. The rudder is actuated by a turn coordination block. Additionally, the platform permits the use of differential elevators for roll control due to the split elevator design. This provides the capability to superpose a fraction of the roll control signal to the elevators, leading to an asymmetric elevator deflection. This measure can be used to increase turn performance. Since the wing folding mechanism permits individual sweep actuation of each wing, the use of asymmetric wing sweep to enhance roll and pitch control has been further studied in [6]. However, this is not used during the flight envelope of this project.

An adaptation of the aerial controller is used for attitude control underwater, also in a cascaded PID fashion. Again, roll and pitch axis are controlled by two independent rate controllers. The main difference from the aerial controller is the lack of a turn coordination. Instead, the yaw angle is not controlled directly but yaw rate is controlled to be zero (yaw is damped) from the moment of dive-in. This measure allows the vehicle to keep a constant azimuth from the moment of dive-in to dive-out. This measure was implemented for reasons of safety during testing and simplicity. During the underwater phase, the vehicle may experience high pitch angles, for example when preparing for dive-out. Avoiding a singular Euler angle Jacobian necessitates switching the global reference frame. Thus, as another adaptation of the underwater controller, the global reference frame is switched automatically to a tailsitter mode when experiencing pitch angles in excess of 60 degrees. Since the wings are folded back during the underwater phase, the use of ailerons to control body roll rate becomes infeasible. Instead, differential elevators are used to control body roll rate. This adapted control mixing

\footnotetext{
${ }^{1}$ http://web.mit.edu/drela/Public/web/avl/

2 https://docs.px4.io/master/en/flight_stack/controller_diagrams.html
} 
maps body roll command to differential elevators and body pitch command to elevator. The actuator commands are then simply superposed for each actuator individually. The control signal mixing was chosen using relative gain array theory to determine the input-output couplings of the control surfaces. Therefore, a simplified, linear relation between elevator deflection and resulting body moment was assumed.

The allocation of roll and pitch commands can be seen in Equation 1. Roll command $\left(Y_{\text {roll }}\right)$ and pitch command $\left(Y_{\text {pitch }}\right)$ are both mapped solely to left and right elevator, $\delta_{E, r}$ and $\delta_{E, l}$ respectively. A nonzero roll command leads to asymmetric elevator deflections.

$$
\begin{aligned}
& \delta_{E, r}=Y_{\text {pitch }}+Y_{\text {roll }} \\
& \delta_{E, l}=Y_{\text {pitch }}-Y_{\text {roll }}
\end{aligned}
$$

\section{EXPERIMENTAL EVALUATION}

Before attempting the full operation cycle (Fig. 11), subsections were tested individually. Starting with basic flight tests, the goal was to achieve stable flight, also in windy conditions.

Unpowered drop tests demonstrated that to fully submerge during dives, the vehicle required the underwater screw to be activated. To test the next phase, the system was fully submerged and commanded to track a reference depth (Fig. 8). To test the critical dive out phase, the system was fixed at a range of pitch angles just below the water surface and then attempted emersion to find the ideal emersion angle (Fig. 9p. In the course of those experiments an angle of $80^{\circ}$ with respect to the water surface proved to be ideal.

The target operation flow can be seen in Fig. 11 The system flies $(a)$ at cruising speed. To dive $(b)$ the wings are folded backwards parallel to the fuselage, as shown in Fig. 10, and the motor changes direction to drive the underwater screw. Once the impact on the water surface is detected through the pressure sensor, the system opens the wings approximately 10 degrees to increase pitch authority and dives to a preset depth $(c)$, where it levels out $(d)$. To emerge, the system pitches upwards $(e)$ and opens the wings fully $(f)$ to reach the water surface at a preset egress angle of approximately 80 degrees. Once the surface is detected by the pressure sensor, the motor is set to full thrust with the aerial propeller and the system pulls itself out of the water $(g)$, giving the pilot time to take control for manual flight. Alternatively the pilot can take over control once the system breaks through the surface, as soon as

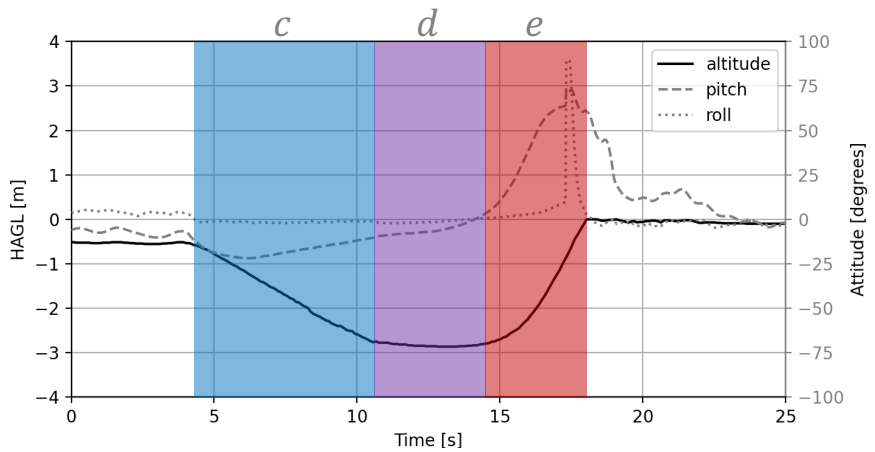

Fig. 8: Height above ground based on data from the pressure sensor. The reference depth $(3 \mathrm{~m})$ can be tracked accurately.

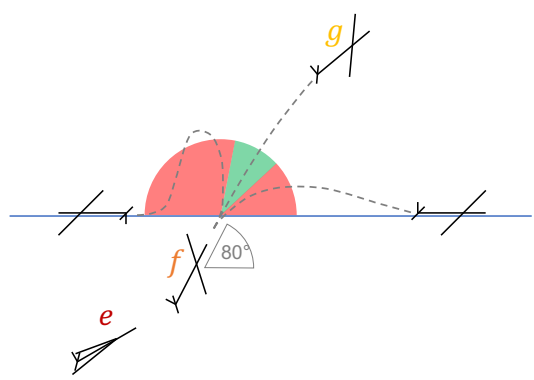

Fig. 9: Testing of diving out angle: Too shallow and the propeller touches the water surface, too steep and the system falls backward. This is due to insufficient control authority at low speeds and the pitch moment induced by the dive out.

connection is established. The necessary precision in timing and control of the whole transition phase is not yet sufficient for a jumping dive-out. Therefore, further investigations into the control of the ESC and the general control of the process are necessary to optimize the propulsion system. Nevertheless the system is capable of transitioning between the two media in less than $2 \mathrm{~s}$, resulting in a dynamic and reliable dive out (success rate above $80 \%$ ), with the most common problem being the direction change of the motor due to issues with measuring the orientation of the rotor with respect to the magnetic field by the ESC in water. In this case the system falls back into the water and can make another dive-out attempt.

To facilitate the testing set up and increase safety, the system started in phase $c$ (underwater). The resulting depth profile measured by the pressure sensor can be seen in Fig. 12 After staying submerged for about 10 seconds, reaching a depth of almost 3 meters, the system rose to the water surface in an angle of 80 degrees and once it broke through the water surface, continued the dive out sequence (Fig. 13).

\section{CONCLUSiON}

\section{A. Discussion}

This paper discusses the design process, construction and experimental evaluation of the Dipper aerial-aquatic unmanned vehicle. The proposed system is capable of flying, diving into water, maneuvering automatically underwater and emerging dynamically back into level flight. The newly developed propulsion concept, based on only one electric motor, allows

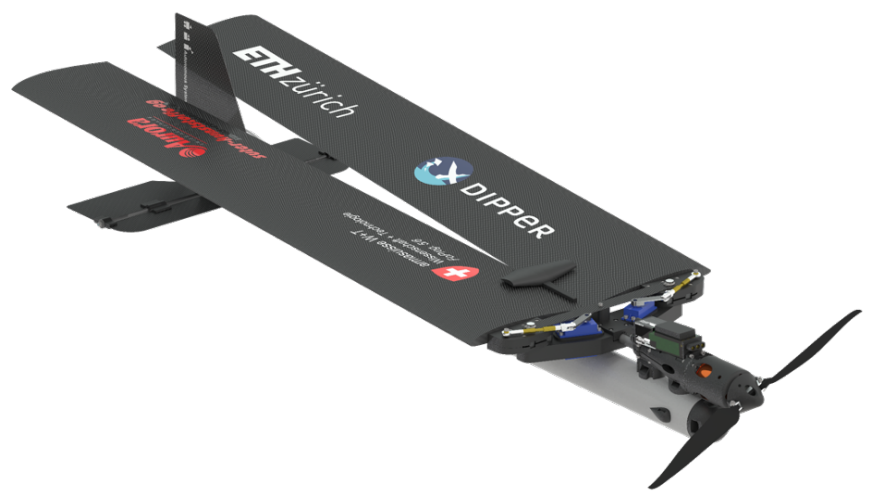

Fig. 10: Diving configuration with the wings and propeller folded backwards. 

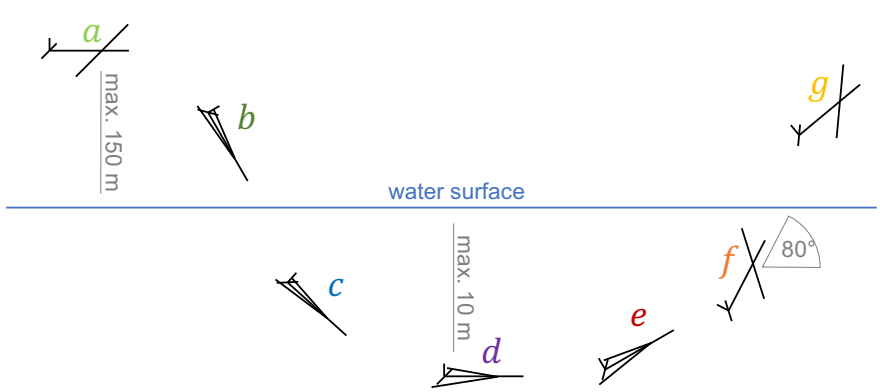

Fig. 11: Full cycle of flight $(a)$, dive $(b, c)$, swim $(d, e)$ and emersion $(f, g)$.
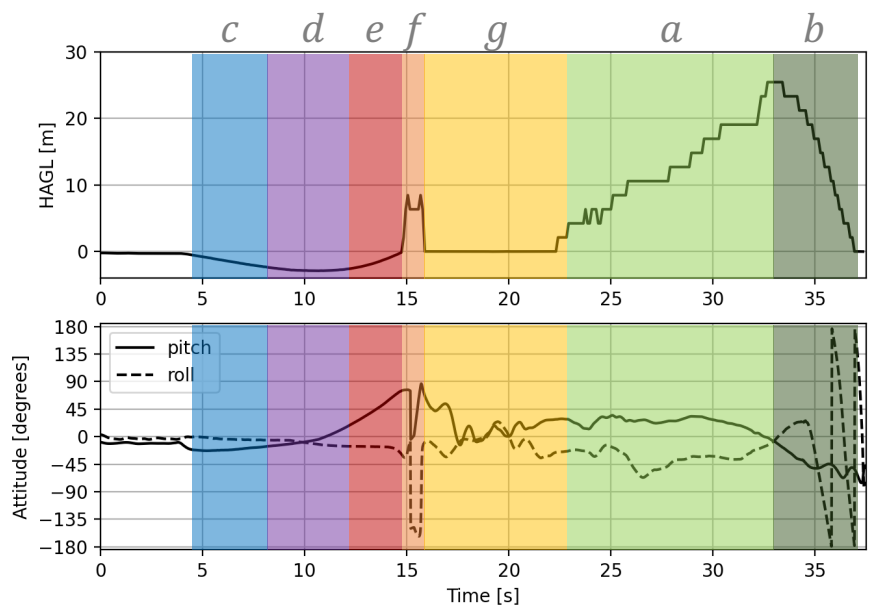

Fig. 12: Height above ground based on data from the pressure sensor The sensor is designed for underwater operation at depths up to 100 $\mathrm{m}$, explaining the discrete steps above the surface $(g, a, b)$, where it offers limited resolution in low density media like air. The pressure peak in $f$ can be explained by unmodelled dynamic effects occurring while breaking through the water surface. During the dive in phase $b$, the system starts spinning after the wings are folded back due to the resulting limited control authority.

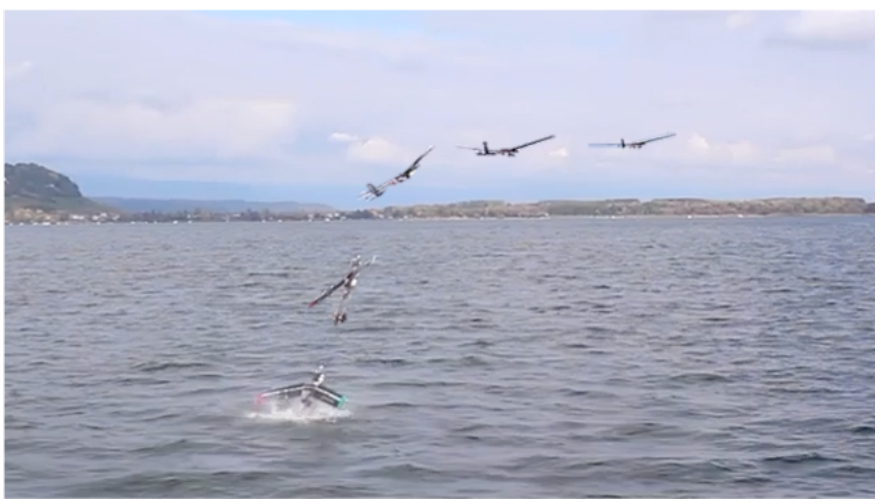

Fig. 13: Emersion phase $(f, g)$ followed by flight $(a)$. highly dynamic locomotion with high efficiency in both media as well as during transition phases. A modular structure allows maintenance and further development to be carried out quickly and easily. The prototype was tested extensively in all phases of operation to ensure robustness and repeatability. It demonstrates the first dynamic, semi-automatic emersion of an aerial-aquatic unmanned vehicle with only one electric motor. Additionally, the system is capable of carrying small sensor payloads like cameras.

\section{B. Future Work}

Enhanced sensor fusion algorithms for underwater operation, a specialized dive out controller and an adapted airspeed sensor, relying on a hot wire anemometer instead of a pitotstatic pressure system, have been developed and implemented to allow the system to perform autonomous missions. However, those systems have yet to be optimized to produce reliable state estimates. Additionally, an ESC with enhanced measuring capabilities would be required to reliably perform jumping dive-outs.

\section{ACKNOWLEDGMENTS}

Support for this work came from armasuisse Science and Technology S+T, Aurora Swiss Aerospace and Suter Kunststoffe AG.

\section{REFERENCES}

[1] H. Alzu'bi, O. Akinsanya, N. Kaja, I. Mansour, and O. Rawashdeh. Evaluation of an aerial quadcopter power-plant for underwater operation. In 10th International Symposium on Mechatronics and its Applications (ISMA), pages 1-4, 2015. doi: 10.1109/ISMA.2015. 7373488.

[2] D. Canelon-Suarez, Y. Wang, and N. Papanikolopoulos. Omnibot: A Small Versatile Robotic Platform Capable of Air, Ground, and Underwater Operation. In 2020 International Conference on Unmanned Aircraft Systems (ICUAS), pages 1742-1747, 2020. doi: 10.1109/ ICUAS48674.2020.9214021.

[3] J. Davenport, R. N. Hughes, M. Shorten, and P. S. Larsen. Drag reduction by air release promotes fast ascent in jumping emperor penguins - a novel hypothesis Marine Ecology Progress Series, 430:171-182, 2011. doi: 10. 3354/meps08868.

[4] P. L. J. Drews, A. A. Neto, and M. F. M. Campos. Hybrid Unmanned Aerial Underwater Vehicle: Modeling and simulation. In 2014 IEEE/RSJ International Conference on Intelligent Robots and Systems, pages 4637-4642, 2014. doi: 10.1109/IROS.2014.6943220.

[5] S. Garthe, S. Benvenuti, and W. A. Montevecchi. Pursuit plunging by northern gannets (Sula bassana) feeding on capelin (Mallotus villosus) Proceedings of the Royal Society of London. Series B: Biological Sciences, 267 (1454):1717-1722, 2000. doi: 10.1098/rspb.2000.1200.

[6] M. Harms, N. Kaufmann, F. M. Rockenbauer, N. Lawrance, T. Stastny, and R. Y. Siegwart. 
Differential Sweep Attitude Control for Swept Wing UAVs. In 2020 International Conference on Unmanned Aircraft Systems (ICUAS), pages 166-175, 2020. doi: 10.1109/ICUAS48674.2020.9214033.

[7] J. Liang, X. Yang, T. Wang, G. Yao, and W. Zhao. Design and experiment of a bionic gannet for plungediving Journal of Bionic Engineering, 10(3):282-291, 2013. doi: 10.1016/S1672-6529(13)60224-3.

[8] M. M. Maia, P. Soni, and F. J. Diez. Demonstration of an Aerial and Submersible Vehicle Capable of Flight and Underwater Navigation with Seamless Air-Water Transition. In XPONENTIAL 2016: An AUVSI Experience, volume 1, pages 700-707, 2016.

[9] R. Siddall and M. Kovač. Launching the AquaMAV: bioinspired design for aerial-aquatic robotic platforms. Bioinspiration \& biomimetics, 9(3):031001, 2014. doi: 10.1088/1748-3182/9/3/031001.

[10] R. Siddall and M. Kovač. A water jet thruster for an aquatic micro air vehicle. In 2015 IEEE International Conference on Robotics and Automation (ICRA), pages 3979-3985, 2015. doi: 10.1109/ICRA.2015.7139755.

[11] R. Siddall, G. Kennedy, and M. Kovač. High-power propulsion strategies for aquatic take-off in robotics In Robotics Research, pages 5-20. Springer, 2018. doi: 10.1007/978-3-319-51532-8_1.

[12] W. Stewart, W. Weisler, M. MacLeod, T. Powers, A. Defreitas, R. Gritter, M. Anderson, K. Peters, A. Gopalarathnam, and M. Bryant. Design and demonstration of a seabird-inspired fixed-wing hybrid UAVUUV system Bioinspiration \& biomimetics, 13(5): 056013, 2018. doi: 10.1088/1748-3190/aad48b.

[13] Y. H. Tan and B. M. Chen. A Lightweight Waterproof Casing for an Aquatic UAV using Rapid Prototyping. In 2020 International Conference on Unmanned Aircraft Systems (ICUAS), pages 1154-1161, 2020. doi: 10.1109/ ICUAS48674.2020.9214029.

[14] Y. H. Tan, R. Siddall, and M. Kovač. Efficient Aerial-Aquatic Locomotion With a Single Propulsion System. IEEE Robotics and Automation Letters, 2(3): 1304-1311, 2017. doi: 10.1109/LRA.2017.2665689.

[15] W. Weisler, W. Stewart, M. B. Anderson, K. J. Peters, A. Gopalarathnam, and M. Bryant. Testing and Characterization of a Fixed Wing Cross-Domain Unmanned Vehicle Operating in Aerial and Underwater Environments. IEEE Journal of Oceanic Engineering, 43(4):969-982, 2018. doi: 10.1109/JOE.2017.2742798. 